\title{
Aspirina en prevención primaria: cuando el árbol no nos deja ver el bosque
}

\author{
Dr. Oscar Bazzino
}

"El mayor enemigo del conocimiento no es la ignorancia, sino la ilusión del conocimiento". Stephen Hawking

Durante el congreso del Colegio Americano de Cardiología, celebrado en marzo de 2019, se presentaron las nuevas guías sobre prevención primaria de enfermedad cardiovascular (CV)(1). En éstas se expresa que debería considerarse la administración de dosis bajas de aspirina (75-100 mg diarios) en adultos seleccionados de entre 40 y 70 años de edad que se encuentran en un nivel alto de riesgo de enfermedad CV, siempre y cuando no presenten una probabilidad elevada de padecer algún tipo de hemorragia. Esta recomendación es de tipo IIb, nivel de evidencia A, que dicho de otra forma significa que existe un margen muy estrecho entre el beneficio y el perjuicio de su utilización.

Hagamos un repaso de la evidencia científica que sostiene esta recomendación. Durante el pasado año 2018 se publicaron tres trabajos muy sólidos que responden a la pregunta sobre qué lugar tiene la aspirina en el ámbito de la prevención primaria: el ASCEND (A Study of Cardiovascular Events in Diabetes trial), el ARRIVE (Aspirin to Reduce Risk of Inicial Vascular Events trial) y el ASPREE (Aspirin in Reducing Events in the Ederly trial).

En el ensayo ASCEND(2) se randomizaron 15.480 diabéticos a recibir $100 \mathrm{mg}$ diarios de aspirina versus placebo. Durante un seguimiento promedio de 7,4 años se produjeron $8,5 \%$ de eventos $\mathrm{CV}$ en el grupo aspirina comparado con $9,6 \%$ en el grupo placebo (RR 0,88; IC 95\%, 0,79-0,97; p = 0,01). Este $12 \%$ de reducción de eventos CV con el uso de dosis bajas de aspirina estuvo contrarrestado por un incremento del orden de $29 \%$ en la tasa de sangrados mayores $(4,1 \%$ con aspirina vs $3,2 \%$ con placebo; RR 1,29; IC 95\%, 1,09-1,52; p=0,003). La mortalidad total fue equivalente entre ambos grupos (RR 0,94; IC 95\%, 0,85-1,04)(3).
En el estudio ARRIVE(4) se comparó a un grupo de personas sin diabetes, con riesgo alto de enfermedad CV, que recibieron aspirina $100 \mathrm{mg}$ diarios, con otro grupo de iguales características que recibieron placebo. La realidad mostró que durante un seguimiento de cinco años los 12.546 participantes que se reclutaron presentaban un riesgo sensiblemente menor al predicto (riesgo bajo a moderado). En este escenario la incidencia del punto final primario (un combinado de infarto de miocardio, accidente cerebrovascular, angina inestable, accidente isquémico transitorio y/o muerte de causa CV) fue $4,3 \%$ en el grupo aspirina, comparado con $4,5 \%$ en el grupo placebo (HR 0,96; IC 95\%, 0,81-1,13; p = 0,6). Al mismo tiempo la incidencia de sangrados de origen gastrointestinal fue el doble en el grupo aspirina comparado con el grupo placebo (HR 2,1; IC 95\%, 1,6-3,28; $\mathrm{p}<0,001$ ). Una vez más, no hubo diferencias significativas entre ambos grupos con respecto a la tasa de eventos hemorrágicos fatales y a mortalidad total (HR 0,99; IC 95\%, 0,80 -1,24; p=0,95).

El estudio ASPREE(5) incluyó a 19.114 individuos mayores de 70 años en prevención primaria que se asignaron aleatoriamente a recibir $100 \mathrm{mg}$ diarios de aspirina o placebo con un seguimiento durante cinco años. No hubo diferencias significativas entre ambos grupos con respecto a la incidencia de enfermedad CV (HR de enfermedad CV del grupo aspirina versus placebo 0,95; IC 95\%, 0,83-1,08). Otra vez la incidencia de sangrados mayores fue significativamente más alta en el grupo aspirina (HR $1,39 ;$ IC $95 \%, 1,18-1,62 ; \mathrm{p}<0,001)$. Un dato que hay que tomar con precaución es el hallazgo de un aumento en la incidencia de mortalidad total del grupo aspirina comparado con el grupo placebo (HR 1,14; IC 95\%, 1,01-1,29).

Como se puede apreciar en los estudios analizados, el beneficio del tratamiento con aspirina en el escenario de la prevención primaria es nulo o muy débil, a tal punto que se ve opacado por el incremento de los eventos hemorrágicos determinando un

Cardiocentro de Asociación Española. Montevideo, Uruguay.

Correspondencia: Dr. Oscar Bazzino. Correo electrónico: obazpe@gmail.com

$\mathrm{El}$ autor declara no tener conflictos de intereses.

Recibido May 29, 2019; aceptado May 30, 2019. 
beneficio clínico neto deficitario. Peor aún, es importante tener en cuenta que en los ensayos contemporáneos se utiliza una definición de infarto de miocardio que incluye eventos isquémicos menores detectados por biomarcadores de alta sensibilidad. Si estos eventos fueran eliminados del punto final primario del ASCEND, el beneficio clínico neto en diabéticos sería más deficitario aún.

Abordando el desafío de la prevención primaria desde otra perspectiva, la utilización de estatinas en este ámbito se asocia a una disminución del riesgo de eventos vasculares mayores; hablamos de una reducción en un orden de $25 \%$ por cada $\mathrm{mmol} / \mathrm{l}$ de descenso del LDL-colesterol (RR con estatinas versus placebo 0,75; IC 95\%, 0,69-0,82)(6). Este benefi-

\section{Bibliografía}

1. Arnett DK, Blumenthal RS, Albert MA, Buroker Esq AB, Goldberger ZD, Hahn EJ, et al. 2019 ACC/AHA Guideline on the Primary Prevention of Cardiovascular Disease: a report of the American College of Cardiology/American Heart Association Task Force on Clinical Practice Guidelines. J Am Coll Cardiol. 2019.pii: S0735-1097(19)33877-X. doi: 10.1016/ j.jacc.2019.03.010

2. Bowman L, Mafham M, Wallendszus K, Stevens W, Buck G, Barton J, et al. Effects of aspirin for primary prevention in persons with diabetes mellitus. N Engl J Med. 2018;379(16):1529-39. doi: 10.1056/NEJMoa1804988

3. Bowman L, Mafhan M, Wallendszus K, Stevens W, Buck G, Barton J, et al. Effects of aspirin for primary prevention in persons with diabetes mellitus. N Engl J Med. 2018 Oct [consulta 29 May 2019];379(16):[aprox.41p.]. Disponible en: https:// www.nejm.org/doi/suppl/10.1056/NEJMoa180498 8/suppl_file/nejmoa1804988_appendix_1.pdf cio se acompaña de un excelente perfil de seguridad y sin las complicaciones hemorrágicas vinculadas a la aspirina. Un dato interesante, es que el porcentaje de participantes que recibieron estatinas en los ensayos ASCEND, ARRIVE y ASPREE fue del orden de $75 \%, 43 \%$ y $34 \%$ respectivamente.

¿Qué recomendación deberíamos hacer a la luz de toda esta evidencia? Bien simple, más allá de una dieta cardiosaludable, la realización de ejercicio físico en forma regular y la cesación del tabaquismo, la mejor estrategia para el uso de aspirina en prevención primaria es prescribir una estatina en su lu$\operatorname{gar}^{(7)}$.

Es cuestión de contemplar el bosque en vez de encandilarnos con el árbol.

Oscar Bazzino, https://orcid.org/0000-0002-0349-6094

4. Gaziano JM, Brotons C, Coppolecchia R, Cricelli $\mathrm{C}$, Darius $\mathrm{H}$, Gorelick PB, et al. Use of aspirin to reduce risk of initial vascular events in patients at moderate risk of cardiovascular disease (ARRIVE): a randomised, double blind, placebo-controlled trial. Lancet 2018; 392(10152):1036-46. doi: 10.1016/S0140-6736(18)31924-X

5. McNeil JJ, Wolfe R, Woods RL, Tonkin AM, Donnan GA, Nelson M, et al. Effect of aspirin oncardiovascular events and bleeding in the healthy elderly. N Engl J Med. 2018;379(16):1509-18.doi: 10.1056/NEJMoa1805819

6. Collins R, Reith C, Emberson J, Armitage J, Baigent C, Blackwell $\mathbf{L}$, et al. Interpretation of the evidence for the efficacy and safety of statin therapy. Lancet 2016; 388: 2532-61.

7. Ridker PM. Should aspirin be used for primary prevention in the post-statin era? N Engl J Med. 2018; 379(16):1572-74. doi: 10.1056/NEJMe1812000 